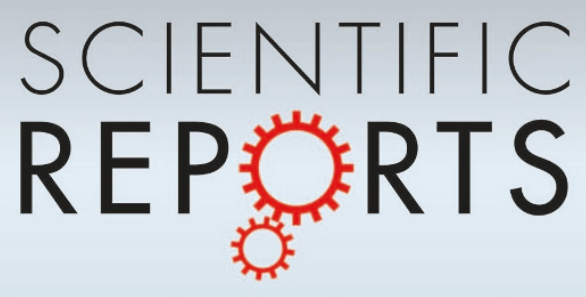

\title{
OPEN \\ Gap plasmon-based metasurfaces for total control of reflected light
}

SUBJECT AREAS:

SUB-WAVELENGTH

OPTICS

NANOPHOTONICS AND

PLASMONICS

SURFACE PATTERNING

TWO-DIMENSIONAL MATERIALS

Received

10 May 2013

Accepted

21 June 2013

Published

8 July 2013

Correspondence and requests for materials should be addressed to A.P. (alp@iti.sdu.dk)

\author{
Anders Pors, Ole Albrektsen, llya P. Radko \& Sergey I. Bozhevolnyi \\ Department of Technology and Innovation, University of Southern Denmark, Niels Bohrs Allé 1, DK-5230 Odense M, Denmark.
}

In the quest to miniaturise photonics, it is of paramount importance to control light at the nanoscale. We reveal the main physical mechanism responsible for operation of gap plasmon-based gradient metasurfaces, comprising a periodic arrangement of metal nanobricks, and suggest that two degrees of freedom in the nanobrick geometry allow one to independently control the reflection phases of orthogonal light polarisations. We demonstrate, both theoretically and experimentally, how orthogonal linear polarisations of light at wavelengths close to $800 \mathrm{~nm}$ can be manipulated independently, efficiently and in a broad wavelength range by realising polarisation beam splitters and polarisation-independent beam steering, showing at the same time the robustness of metasurface designs towards fabrication tolerances. The presented approach establishes a new class of compact optical components, viz., plasmonic metasurfaces with controlled gradient birefringence, with no dielectric counterparts. It can straightforwardly be adapted to realise new optical components with hitherto inaccessible functionalities.

$\mathrm{n}$ the ongoing quest to miniaturise photonics to the micro- and nanoscale, conventional optical components, such as dielectric lenses and polarisers, are no longer adequate for steering and manipulating light owing to their inherently bulky nature. One of currently investigated approaches to resolve this issue is the application of metamaterials, which allow one to design materials with specific and exotic optical properties, including negative refraction ${ }^{1,2}$ and moulding the flow of light using inhomogeneous metamaterials and transformation optics $^{3,4}$.

An alternative approach towards light control at the nanoscale, which also seems more feasible for practical realisation and implementation, is concerned with two-dimensional (2D) metal nanostructures with subwavelength periodicity, also known as metasurfaces. Despite having negligible thicknesses as compared to the incident wavelength, metasurfaces are characterised by the ability to strongly manipulate both the amplitude and phase of impinging light near (plasmonic) resonances of the unit cell constituents ${ }^{5}$. It should be however noted that resonant elements allow one to control the phase only in a limited range, from 0 to $\pi$ (radians), due to their Lorentz-like polarisabilities ${ }^{6-9}$. In order to access the whole phase space of $2 \pi$, two different approaches are typically employed. In the first approach, the $2 \pi$-space is achieved with transmitted/reflected light polarised orthogonally to the incident wave by using $\mathrm{V}$-shaped nanoantennas that can be designed to realise a variety of flat optical components ${ }^{10-15}$. Nevertheless, the cross-polarisation condition sets an upper bound to the efficiency of the devices that in the limit of negligible absorption is theoretically estimated to $25 \%{ }^{16}$. Importantly, all demonstrated functionalities can also be realised, in principle, by profiling the surface of a dielectric substrate, e.g., with ion-beam milling technique, in the fashion employed for blazed gratings ${ }^{17}$. The second approach is similar, at least from the first sight, to that used in reflectarray antennas ${ }^{18}$, in which an array of metal rectangular patches is placed in close proximity to a metal film or substrate, only separated by an optically thin dielectric spacer. The metalbacked design ensures an almost full control of the phase space together with a high efficiency, reaching $100 \%$ in the limit of negligible Ohmic losses. These two attractive properties have been demonstrated in the realisation of efficient wave plates ${ }^{19-21}$, focusing mirrors ${ }^{22,23}$, blazed gratings ${ }^{24}$, and components interfacing propagating and surface waves $^{25}$. In the aforementioned work, the metasurface functionalities are related to a proper design of the phase profile for one polarisation, mimicking the phase accumulation acquired when light transverses conventional optical components. Metal-backed metasurfaces, however, seem to be much more versatile according to very recent numerical simulations of metasurfaces showing polarisation-sensitive reflection in the near-infrared ${ }^{24}$ and infrared ${ }^{26}$ regimes. Note, though, that a design technique and experimental verification of such gradient birefringent metasurfaces are still lacking.

Here, we elucidate the underlying physics responsible for operation of metal-backed metasurfaces in visible and near-infrared, emphasising the importance of excitation of gap surface plasmons (GSP) under metal patches or nanobricks ${ }^{20,21,23}$. The developed physical understanding allows us to generalise the design rules of plasmonic, 
a

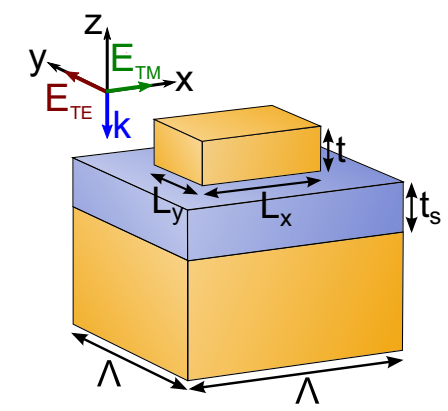

b

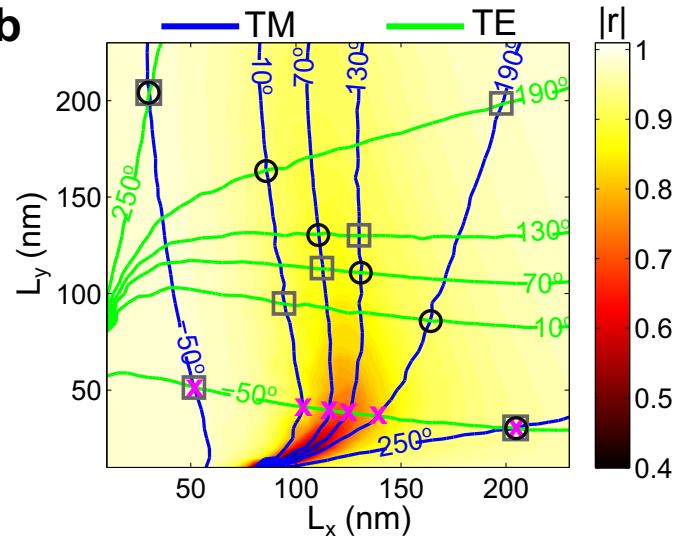

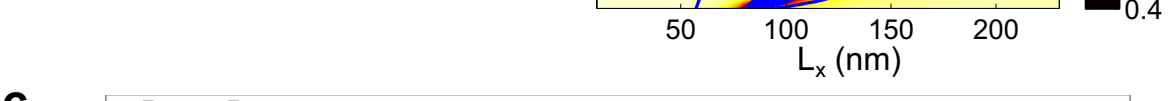
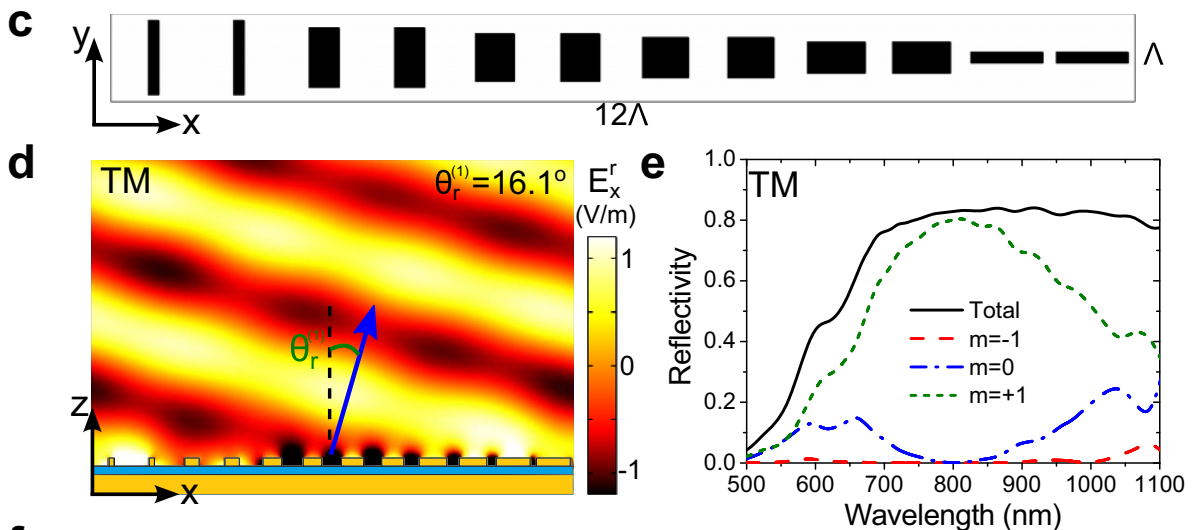

f
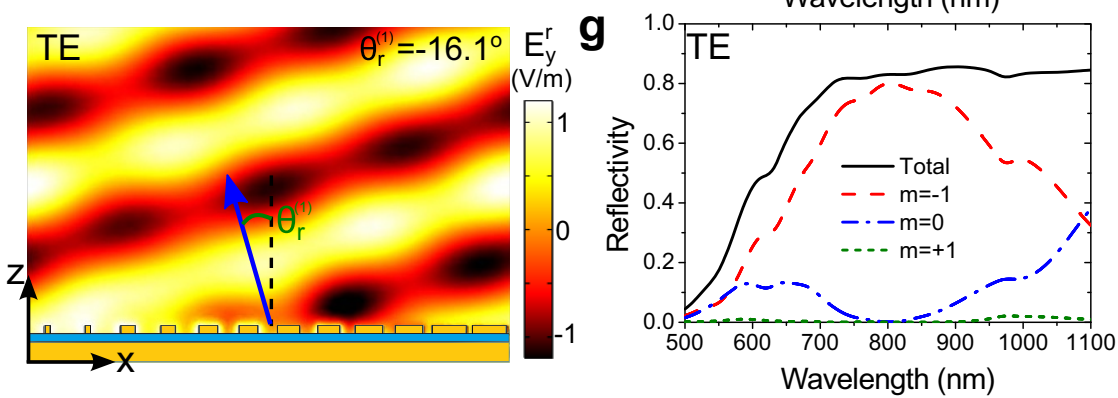

Figure $1 \mid$ Independent control of TE and TM polarisation. (a) Sketch of basic unit cell consisting of a gold nanobrick on top of a glass spacer and gold substrate. (b) Calculated reflection coefficient $r$ as a function of nanobrick widths for $\Lambda=240 \mathrm{~nm}, t=t_{s}=50 \mathrm{~nm}$, and $\lambda=800 \mathrm{~nm}$ (for further details see Supporting Information). Colour map shows the reflection coefficient amplitude for TM polarisation, while lines are contours of the reflection phase for both TM and TE polarisation. (c) Super cell of metasurface functioning as a polarisation beam splitter (case 1): TM (TE) waves are reflected into $+1(-1)$ diffraction order. $(\mathrm{d}, \mathrm{e})$ Theoretical performance of the metasurface for TM polarisation, displaying $(\mathrm{d})$ the $\mathrm{x}$-component of reflected E-field $\left(E_{x}^{r}\right)$ just above the metasurface at the design wavelength (the amplitude of incident E-field is $1 \mathrm{~V} / \mathrm{m}$ ), and (e) amount of incident light reflected into the lowest diffraction orders $m$ as a function of wavelength. $(f, g)$ Performance of the metasurface for TE polarisation.

viz. GSP-based, metasurfaces and demonstrate, both theoretically and experimentally, that orthogonal linear polarisations of reflected light can be manipulated independently, efficiently and in a broad wavelength range by exploiting two degrees of freedom (DOF) in the nanobrick geometry.

\section{Results}

Let us start by considering a GSP-based metasurface in a gold-glassgold configuration with the top layer representing a 240 -nm-period array of nanobricks illuminated at normal incidence (Fig. 1a). The nanobrick dimensions $L_{x}$ and $L_{y}$ represent the two DOF that we are going to exploit, leaving all other system parameters constant (note that the lower gold part is considered optically thick). Considering the complex reflection coefficient for two orthogonal polarisations (denoted as TM and TE for the incident light being polarised along the $\mathrm{x}$ - or $\mathrm{y}$-axis, respectively), we find immediately that, near the GSP resonance, the two DOF allow us to independently vary the phase of reflected polarisations practically within the whole phase space of $2 \pi$ (Fig. 1b). In view of that, it is evident that full control of the reflection phase requires the GSP resonance to exist within the parameter space $\left(L_{x}\right.$ and $\left.L_{y}\right)$, otherwise, limited by the unit cell period $\Lambda$. The spacing between equidistant contour lines of the phase and the extent of amplitude variation are dependent on the choice of geometrical parameters, and can be directly related to the properties of GSPs as follows. Close to the GSP resonance that is characterised by strong absorption of the incident light (Supplementary Fig. S1), the phase varies rapidly over the range of $\pi$ as expected for the Lorentz-like response of an oscillator (Fig. 1b). Away from the resonance, the reflection phase is influenced by the retardation of the incident wave when being reflected primarily by the continuous gold layer (for small-area nanobricks) and by the processes of in- and out-coupling of the incident radiation to GSPs (for large-area nanobricks). Finally, for very narrow nanobricks (in the direction perpendicular to their propagation), the GSP gradually transforms into a surface plasmon 
mode supported by a nanorod with the propagation constant increasing rapidly for smaller widths, as seen in bending of the phase contours and absorption locations towards shorter lengths (Fig. 1b and Supplementary Fig. S2).

In order to demonstrate the total control of reflected light with GSP-based metasurfaces, we now design three gradient metasurfaces that, in all cases, anomalously reflect TM waves in the same direction, while the reflection direction for TE waves is engineered at will. According to the equivalence between the generalised laws of refraction and reflection and diffraction theory, the designed metasurfaces may also be viewed as flat blazed gratings, where the angle of anomalous reflection is equal to the first order diffraction angle ${ }^{17}$. Importantly, these blazed gratings should operate independently (and differently) for different polarisations, a functionality that is not accessible with conventional approaches. Using the grating terminology, a polarisation beam splitter (case 1) that reflects TM and TE waves into \pm 1 diffraction order, respectively, represents a metasurface with a linear phase gradient whose sign is opposite for the two polarisations. In this case, our goal is to implement the reflection coefficient $r(x)=A \exp \left( \pm i 2 \pi x / \Lambda_{s c}\right)$, where $A \leq 1$ is the amplitude constant, $\Lambda_{s c}$ is the (super cell) grating periodicity, $x$ is the spatial coordinate, and the sign of the phase gradient depends on the polarisation. Such a design can be derived from Fig. 1b, where the reflection phase for both TM and TE polarisation is discretised into six constant-phase contour lines with a $60^{\circ}$ step, and the appropriate nanobrick dimensions correspond to the intersections marked with circles. Note how this visual solution of the appropriate nanobricks contrasts to the search algorithm used in the design of birefringent metasurfaces in the infrared regime ${ }^{26}$. The corresponding metasurface can be constructed by sequentially placing the six elements with the center-to-center distance of $\Lambda=240 \mathrm{~nm}$ into a super cell (Fig. 1c), with these elements being arranged in pairs in order to lower the first order diffraction angle $\theta_{r}^{(1)}$ to $16.1^{\circ}$. Note that the described approach is based on the assumption that the coupling between neighbouring elements is weak, a fact that we verified by numerical modelling (see Supplementary Fig. S2). The cost of the increased super cell period is a more apparent stair-casing of the linear phase profile (Fig. 1d,f), which results in a slightly decreased performance of the metasurface (Supplementary Fig. S3). Numerical simulations of the designed gradient metasurface show (Fig. 1e,g) that practically all reflected light for TM (TE) polarisation is contained within the $+1(-1)$ diffraction order at the design wavelength of $800 \mathrm{~nm}$, with the total reflectivity being limited to $\sim 80 \%$ because of Ohmic losses in the metal.

The selection of nanobrick dimensions for the independent phase control of orthogonal polarisations relies on intersection of the corresponding constant-phase lines for TM and TE polarisations, which is not perfect as, e.g., the $250^{\circ}$-phase lines do not intersect (Fig. 1b). Ideally, one would also prefer to achieve a constant reflection amplitude, but with the two DOF in our geometry it is possible to adjust only two wave parameters - the reflection phases of two polarisations or the phase and amplitude of one polarisation ${ }^{23}$. On the other hand,

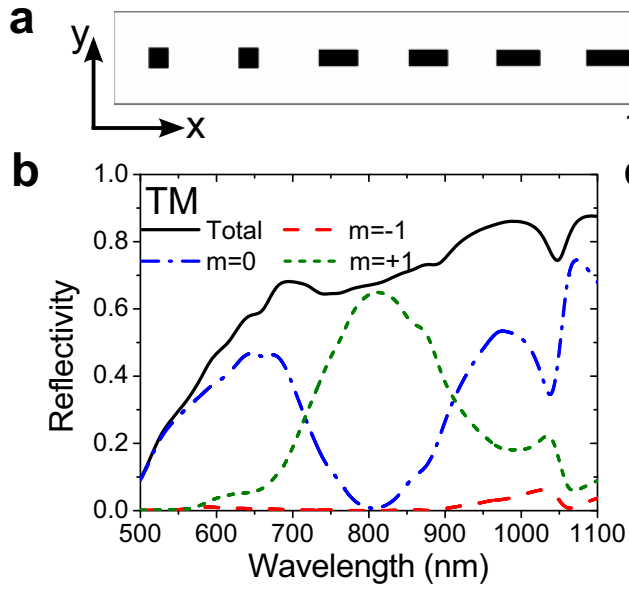

$12 \wedge$
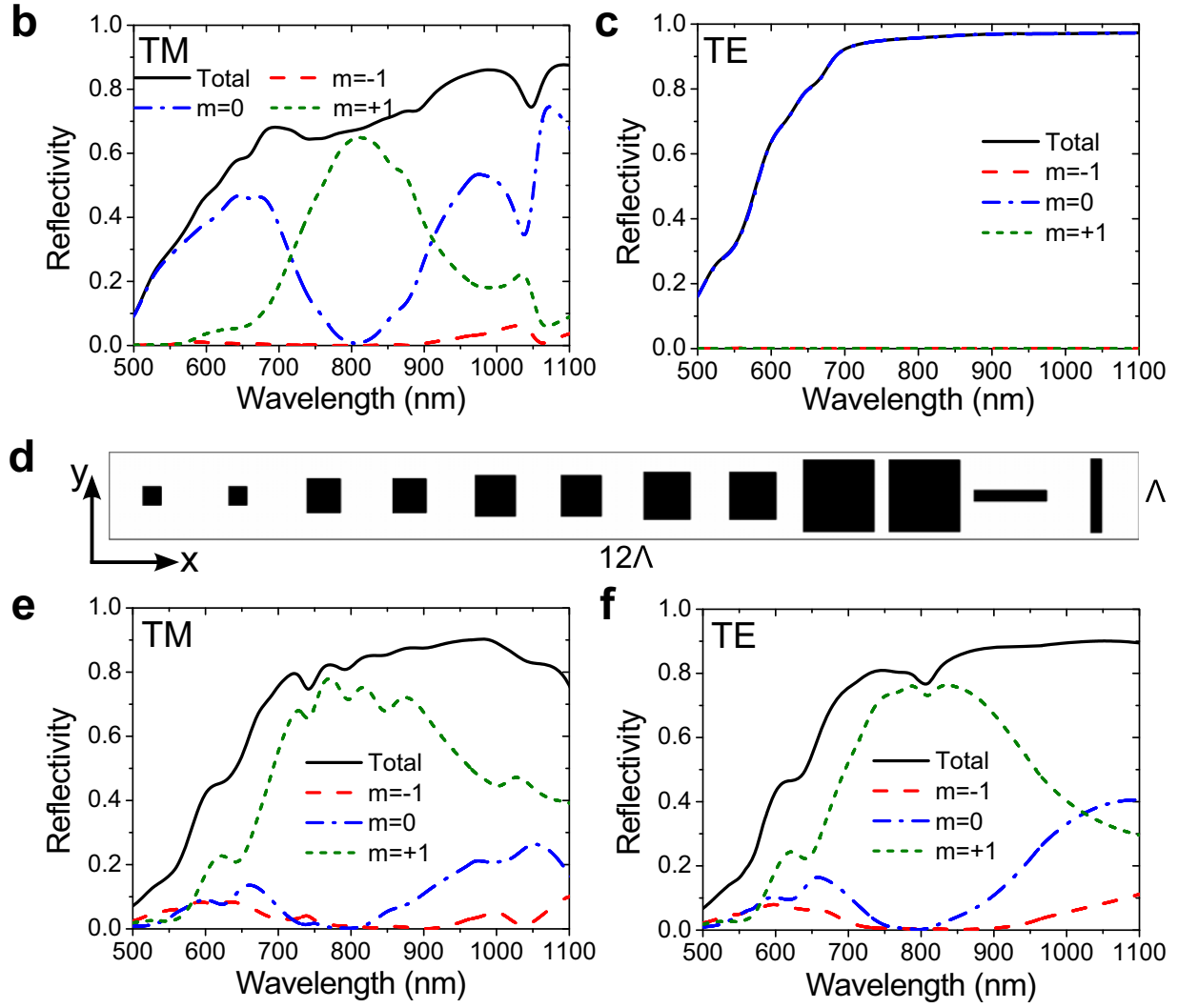

Figure $2 \mid$ Functionalised gradient GSP-metasurfaces. (a-c) Metasurface polarisation beam splitter (case 2): TM (TE) waves are reflected into +1 (0) diffraction order. (d-f) Polarisation-independent beam steering (case 3): TM and TE waves are reflected into +1 diffraction order. (a,d) Top-view of designed super cells. (b,e) Theoretical performance of the metasurfaces for TM polarisation, displaying the amount of incident light reflected into the lowest diffraction orders $m$ as a function of wavelength. (c,f) Performance of the metasurfaces for TE polarisation. 

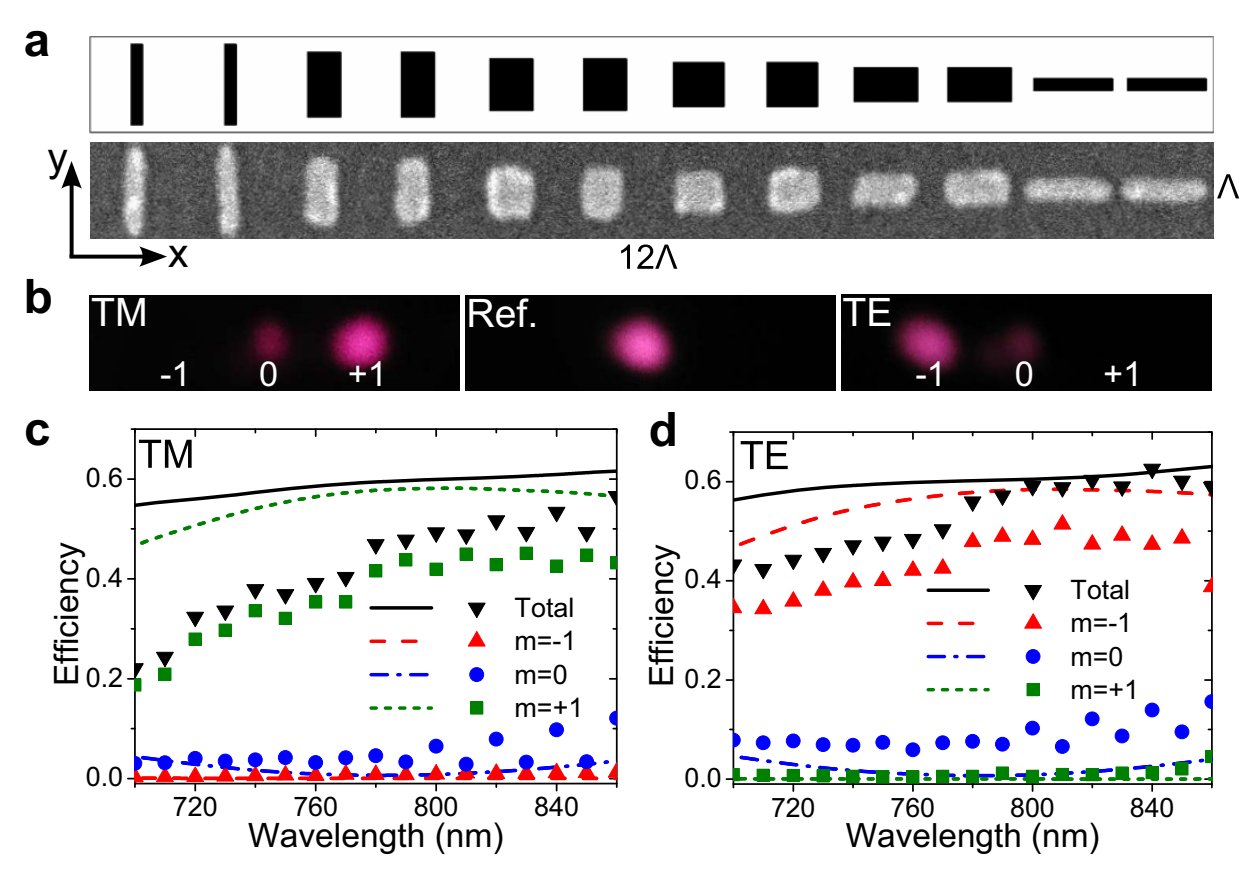

\section{$12 \wedge$}

b TM

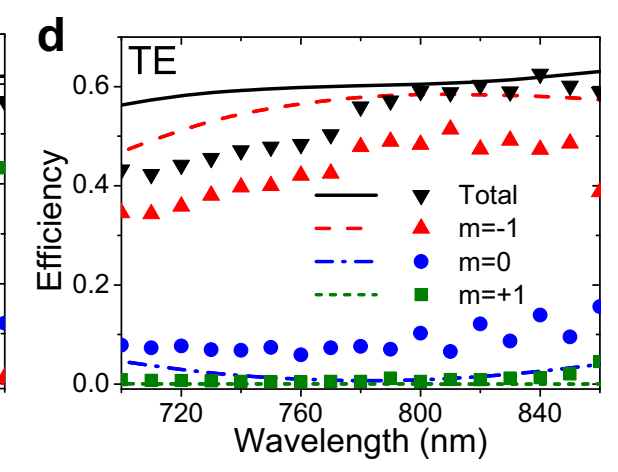

Figure 3 Experimental verification of polarisation beam splitter (case 1): TM (TE) waves are reflected into $+1(-1)$ diffraction order.

(a) Representative scanning electron microscopy (SEM) image of fabricated super cell. (b) Optical images of the diffraction spots at $\lambda=800 \mathrm{~nm}$ for TM and TE polarisation, and reference sample (Ref.) consisting of a $100 \mathrm{~nm}$ gold film overlaid by $50 \mathrm{~nm}$ of silicon dioxide. (c,d) Measured (markers) and calculated (lines) diffraction efficiencies for orders $|m| \leq 1$ for (c) TM and (d) TE polarisation.

the equivalence in operation of gradient metasurfaces and blazed gratings suggests that the presented approach should be robust against (design and fabrication) imperfections ${ }^{17}$, an important feature which becomes also apparent in the following consideration of other two cases. Keeping TM waves being diffracted into +1 order, we now design metasurfaces that would reflect TE waves specularly (case 2 ) or into +1 diffraction order (case 3 ). Note that the latter case corresponds to the polarisation-independent anomalous reflection (beam steering). The two different configurations correspond to nanobrick dimensions determined by the constant-phase line intersections in Fig. 1b marked with crosses and squares, respectively. Clearly, the performance of case 2 for TM waves is affected by considerable variation in the reflection amplitude for different elements, whereas case 3 suffers from the absence of a nanobrick ensuring the reflection phase of $250^{\circ}$ for both polarisations. Nevertheless, the designed metasurfaces in both cases perform well when being simulated as seen in Fig. 2. Note that, in case 3, instead of the pair of equivalent nanobricks, we used two different $250^{\circ}$-phase nanobricks selected separately for TM and TE waves (Fig. 2d). Regarding case 2, it is clearly seen how TM waves are almost exclusively reflected into first diffraction order with a theoretical reflectivity of $\sim 70 \%$ at the design wavelength (Fig. 2b). For TE polarisation, on the other hand, the incident light barely interacts with the nanobricks, which lead to a reflectivity resembling that of a plane gold substrate (Fig. 2c). For case 3 , it is evident how both TM and TE waves are anomalously reflected in +1 diffraction order, while both polarisations demonstrate a broadband response with reflectivity of $\sim 75 \%$ at $\lambda=$ $800 \mathrm{~nm}$ (Fig. 2e,f). The difference between reflection into first diffraction order and the total reflectivity, which is especially apparent for TM polarisation (Fig. 2e), is due to reflection into higher diffraction orders not displayed in Fig. 2.

With the above numerical simulations illustrating the possibility to independently and efficiently control reflected light of orthogonal polarisation using gradient GSP-metasurfaces, we now move on to the experimental verification. Figures 3-5 display representative images of lithographically fabricated super cells, optical images of diffraction spots, and diffraction efficiencies for study case 1-3, respectively. In all cases, reasonable correlation is found between designed and fabricated super cells (Fig. 3a, 4a, 5a, and Supplementary Fig. S4), though discrepancies are also clearly visible. For example, in case 3 , one notices how the fifth pair of elements in the super cell is connected to neighbouring elements along the $y$ directions (Fig. 5a). Nonetheless, the designed metasurfaces are robust towards fabrication imperfections, as evident from the optical images of the reflected light, demonstrating the independent steering of orthogonal polarisations (Fig. 3b, $4 \mathrm{~b}$, and 5b). Moreover, the performance of the GSP-metasurfaces is further quantified by the determination of the zero and first order diffraction efficiencies (Fig. 3c,d, $4 c, d$, and $5 c, d)$. The measurements are compared with numerical calculations (lines) for which we use the designed dimensions of super cells and only one fitting parameter: the imaginary part of the gold permittivity is increased by four times to account for surface scattering and grain boundary effects in thin films as well as the increased damping associated with the 3-nm-thin Ti adhesion layers between gold-glass interfaces ${ }^{21}$. In general, we observe a reasonable agreement between the measured and calculated diffraction efficiencies, verifying the independent and broadband control of TM and TE waves, albeit with a reduced efficiency of $\sim 50 \%$ near the design wavelength (theory: $\sim 80 \%$, c.f. Fig. 1 and 2 ) related to the just mentioned loss channels. Measurements also show a non-vanishing zero order diffraction, a feature that we ascribe to the imperfections in the fabricated metasurfaces.

\section{Discussion}

We have illustrated with the examples in the previous section how the GSP-based metasurfaces with two DOF can be designed to independently, efficiently and in a broadband wavelength range control the phase of reflected TE and TM waves, demonstrating also the practical feasibility and robustness of the presented approach. Although the examples were limited to gradient metasurfaces with variation along the $\mathrm{x}$-axis, it should be stressed that the reflection coefficients depending on two spatial coordinates can be just as easily implemented, thus opening up a wide range of possible functionalities, including but not limited to anomalous reflection of TM and TE 


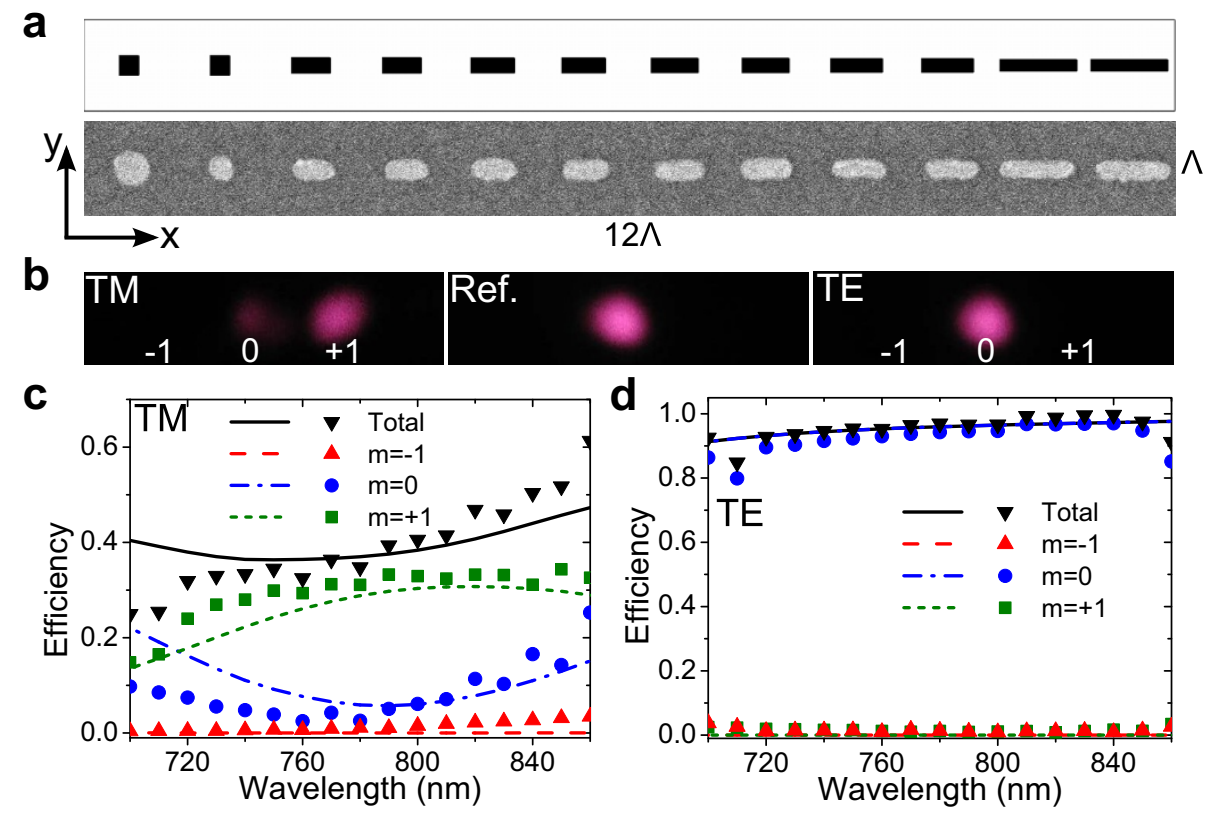

Figure 4 | Experimental verification of independent control of TM and TE waves (case 2): TM (TE) waves are reflected into +1 (0) diffraction order. (a) Comparison of designed and representative SEM image of fabricated super cell. (b) Optical images of the diffraction spots for TM and TE polarisation, and reference sample (Ref.) at $\lambda=800 \mathrm{~nm}$. (c,d) Measured (markers) and calculated (lines) diffraction efficiencies for orders $|m| \leq 1$ for TM and TE polarisation, respectively.

waves in two orthogonal planes, polarisation beam-splitting combined with strong focusing, and linear-to-radial (or azimuthal) polarisation conversion. Furthermore, we stress that the shortcomings of the nanobrick design, especially apparent in study case 3, can be alleviated with more complex elements with a larger number of design parameters - one such element is the nanocross (Supplementary Fig. S5). Finally, we would like to emphasise that the manipulation of two wave parameters alternatively allow for the control of both the amplitude and phase of reflected light for one polarisation, a feature that was already used in our design of focusing metasurfaces to ensure the same reflection amplitude ${ }^{23}$. This idea can be extended further and employed to obtain a practically arbitrary distribution of phase and amplitude of reflected (linearly polarised) light. For example, one can design a metasurface acting as a beam splitter that splits the incident light into \pm 1 diffraction order by implementing the reflection coefficient $r=A \sin \left(2 \pi / \Lambda_{s c} x\right)$. In this
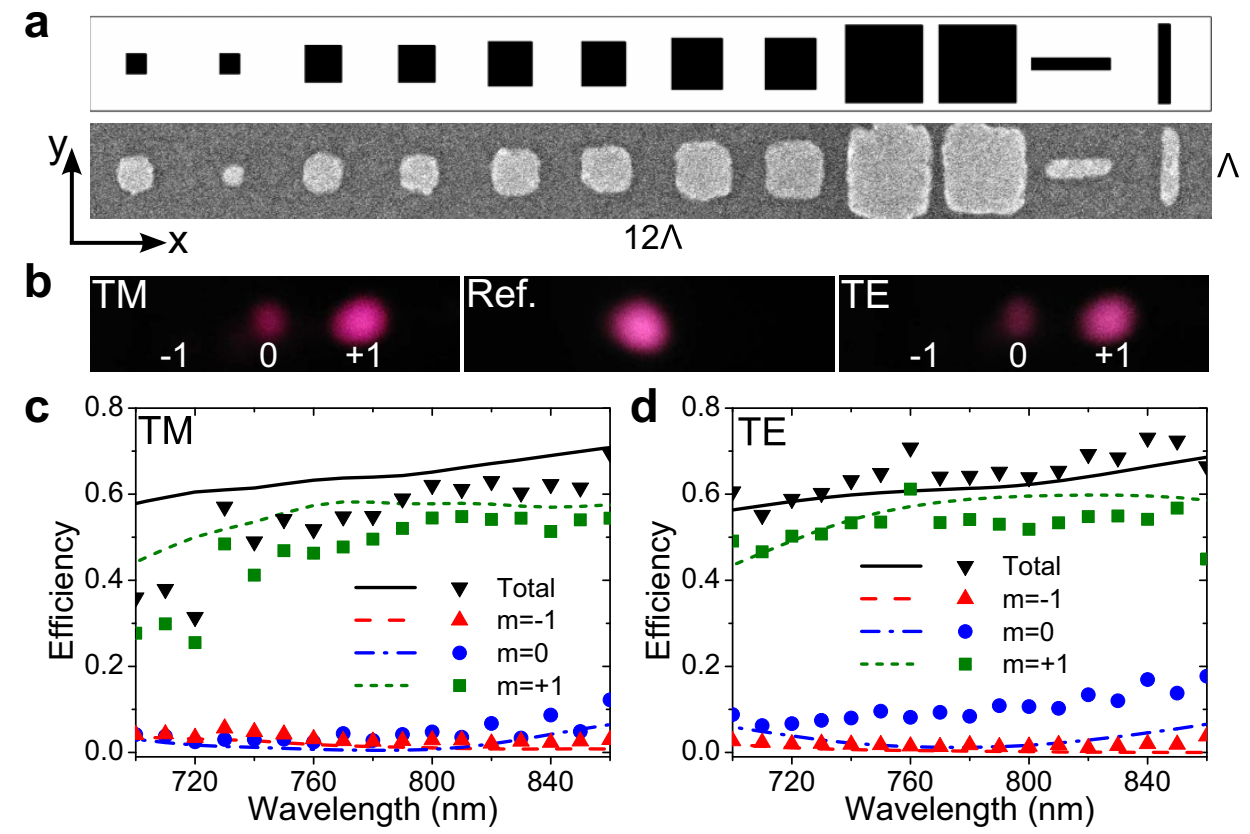

$12 \wedge$

TE
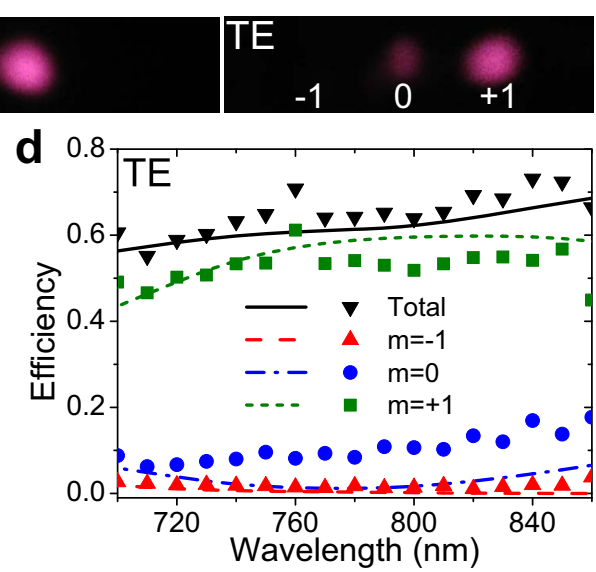

Figure 5 Experimental verification of polarisation-independent blazed grating (case 3): TM and TE waves are reflected into +1 diffraction order. (a) Comparison of designed and representative SEM image of fabricated super cell. (b) Optical images of the diffraction spots for TM and TE polarisation, and reference sample (Ref.) at $\lambda=800 \mathrm{~nm}$. (c,d) Measured (markers) and calculated (lines) diffraction efficiencies for orders $|m| \leq 1$ for $\mathrm{TM}$ and TE polarisation, respectively. 
case, one should make use of the strong variation in the metasurface reflection amplitude close to the GSP resonance (Fig. 1b); a region that otherwise normally belongs to design of plasmonic super absorbers $^{27}$. One should also expect that half of the incident light will have to be absorbed in this case (Supplementary Fig. S6).

Overall, the presented approach establishes a new class of compact optical components, viz., plasmonic metasurfaces with controlled gradient birefringence, that has no dielectric counterparts. It can straightforwardly be adapted to realise new optical components with hitherto inaccessible functionalities as well as extended to other wavelength ranges. We believe that the robustness, efficiency and scalability of the considered configurations will stimulate the development of novel planar optical components compatible with and easily integrated in nanophotonic systems.

\section{Methods}

Modelling. All modelling results are performed using the commercial available finite element software Comsol Multiphysics, ver. 4.3a. In the calculations, it is only necessary to model a single unit cell or super cell by applying periodic boundary conditions on the vertical sides of the cell. The incident wave is in all cases assumed to be a plane wave propagating normal to the surface with either TE or TM polarisation (see Fig. 1a). In our gold-glass-gold configurations, the permittivity of gold is described by interpolated experimental values ${ }^{28}$ while glass, assuming to be silicon dioxide, takes on the constant refractive index $n=1.45$. The medium above the nanobricks is chosen to be air. The air domain is truncated using perfectly matched layers to minimize reflections, while a perfect electric conductor boundary condition is applied on the bottom side of the optically thick gold substrate. Regarding the complex reflection coefficients of the GSP-based metasurfaces (Fig. 1b and Supplementary Fig. S2), the phase is determined at the top surface of the nanobricks. Experimental measured diffraction efficiencies are compared with calculated values (Fig. 3-5). In these calculations the imaginary part of the gold permittivity is increased by a factor of four to take into account additional losses related to surface scattering and grain boundary effects in thin films as well as the increased damping associated with the $3 \mathrm{~nm}$ Ti adhesion layers between gold-glass interfaces ${ }^{21}$. The diffraction efficiencies are then obtained by calculating the reflection into the different diffraction orders and normalising with the reflectivity from the reference configuration (50 $\mathrm{nm}$ silicon dioxide on top of a gold substrate).

Fabrication. The GSP-based metasurfaces are fabricated using electron-beam lithography and lift-off technique. On a base substrate of silicon, $3 \mathrm{~nm}$ of titanium, $100 \mathrm{~nm}$ of gold and $3 \mathrm{~nm}$ of titanium are sequentially deposited by E-beam evaporation and subsequently $50 \mathrm{~nm}$ of $\mathrm{SiO}_{2}$ is deposited by RF-sputtering in a Cryofox 600 system equipped with a Temescal SuperSource2 E-beam source and a MAK magnetron RF-sputter source. Following a spin coating of $120 \mathrm{~nm}$ PMMA $950 \mathrm{~K} \mathrm{A2}$, the gold nanobrick structures with an interparticle distance of $240 \mathrm{~nm}$ are lithographically defined by E-beam lithography and formed by E-beam evaporation of $3 \mathrm{~nm} \mathrm{Ti}$ and $50 \mathrm{~nm}$ gold followed by a lift-off process. One should note that the three layers of $3 \mathrm{~nm}$ titanium are used as adhesion layers at the silicon-gold and glassgold interfaces. The nanobricks are arranged in $28.8 \mu \mathrm{m} \times 28.8 \mu \mathrm{m}$ arrays comprised of 10 and 100 super cells in the $\mathrm{x}$ - and $\mathrm{y}$-direction, respectively, and they are exposed at $30 \mathrm{kV}$ using a JEOL-640LV electron microscope equipped with an ELPHY Quantum lithography system. The resulting structures are imaged using a Hitachi S4800 field emission scanning electron microscope.

Optical characterization. Experimental characterisation of the fabricated structures is performed by directly measuring the intensity of the anomalously reflected laser beam into various diffraction orders $(0$ and \pm 1$)$. A low-power $(\sim 5 \mathrm{~mW})$ continuouswave laser beam from Spectra-Physics 3900 S Ti:Sapphire laser, tunable in the wavelength range $700-860 \mathrm{~nm}$, was weakly focused using a $5 \times$ objective into a spot with diameter $\sim 20 \mu \mathrm{m}$, so that it was illuminating an array of gold nanobricks, not extending beyond them. A beam splitter right before the surface of the sample allowed separating the incident and the reflected signals and measuring the intensity of the latter, which is then normalised to the intensity of the beam reflected from a smooth surface without nanobricks. In order to visualise the effect, we also projected the reflected pattern onto a charge-coupled device camera (Fig. 3b, 4b, and 5b).

1. Smith, D. R., Padilla, W. J., Vier, D. C., Nemat-Nasser, S. C. \& Schultz, S. Composite medium with simultaneously negative permeability and permittivity. Phys. Rev. Lett. 84, 4184-4187 (2000).

2. Shelby, R. A., Smith, D. R. \& Schultz, S. Experimental verification of a negative index of refraction. Science 292, 77-79 (2001)

3. Pendry, J. B., Schurig, D. \& Smith, D. R. Controlling electromagnetic fields. Science 312, 1780-1782 (2006)

4. Schurig, D. et al. Metamaterial electromagnetic cloak at microwave frequencies. Science 314, 977-980 (2006).
5. Zhao, Y., Engheta, N. \& Alú, A. Homogenization of plasmonic metasurfaces modeled as transmission-line loads. Metamaterials 5, 90-96 (2011).

6. Pors, A. et al. Plasmonic metamaterial wave retarders in reflection by orthogonally oriented detuned electrical dipoles. Opt. Lett. 36, 1626-1628 (2011).

7. Zhao, Y. \& Alú, A. Manipulating light polarization with ultrathin plasmonic metasurfaces. Phys. Rev. B 84, 205428 (2011).

8. Roberts, A. \& Lin, L. Plasmonic quarter-wave plate. Opt. Lett. 37, 1820-1822 (2012).

9. Lin, L., Goh, X. M., McGuinness, L. P. \& Roberts, A. Plasmonic lenses formed by two-dimensional nanometric cross-shaped aperture arrays for fresnel-region focusing. Nano Lett. 10, 1936-1940 (2010).

10. Yu, N. et al. Light propagation with phase discontinuities: generalized laws of reflection and refraction. Science 334, 333-337 (2011).

11. Ni, X., Emani, N. K., Kildishev, A. V., Boltasseva, A. \& Shalaev, V. M. Broadband light bending with plasmonic nanoantennas. Science 335, 427 (2012).

12. Aieta, F. et al. Out-of-plane reflection and refraction of light by anisotropic optical antennas metasurfaces with phase discontinuities. Nano Lett. 12, 1702-1706 (2012).

13. Aieta, F. et al. Aberration-free ultrathin flat lenses and axicons at telecom wavelengths based on plasmonic metasurfaces. Nano Lett. 12, 4932-4936 (2012).

14. Yu, N. et al. A broadband, background-free quarter-wave plate based on plasmonic metasurfaces. Nano Lett. 12, 6328-6333 (2012).

15. Chen, X. et al. Dual-polarity plasmonic metalens for visible light. Nat. Commun. $\mathbf{3}$, 1198 (2012).

16. Monticone, F., Estakhri, N. M. \& Alú, A. Full control of nanoscale optical transmission with a composite metascreen. Phys. Rev. Lett. 110, 203903 (2013).

17. Larouche, S. \& Smith, D. R. Reconciliation of generalized refraction with diffraction theory. Opt. Lett. 37, 2391-2393 (2012).

18. Pozar, D. M., Targonski, S. D. \& Syrigos, H. D. Design of millimeter wave microstrip reflectarrays. IEEE Trans. Antennas Propag. 45, 287-296 (1997)

19. Hao, J. et al. Optical metamaterial for polarization control. Phys. Rev. A 80, 023807 (2009).

20. Pors, A. \& Bozhevolnyi, S. I. Efficient and broadband quarter-wave plates by gapplasmon resonators. Opt. Express 21, 2942-2952 (2013).

21. Pors, A., Nielsen, M. G. \& Bozhevolnyi, S. I. Broadband plasmonic half-wave plates in reflection. Opt. Lett. 38, 513-515 (2013).

22. Li, X. et al. Flat metasurfaces to focus electromagnetic waves in reflection geometry. Opt. Lett. 37, 4940-4942 (2012).

23. Pors, A., Nielsen, M. G., Eriksen, R. L. \& Bozhevolnyi, S. I. Broadband focusing flat mirrors based on plasmonic gradient metasurfaces. Nano Lett. 13, 829-834 (2013).

24. Sun, S. et al. High-efficiency broadband anomalous reflection by gradient metasurfaces. Nano Lett. 12, 6223-6229 (2012).

25. Sun, S. et al. Gradient-index meta-surfaces as a bridge linking propagating waves and surface waves. Nat. Mater. 11, 426-431 (2012).

26. Farmahini-Farahani, M. \& Mosallaei, H. Birefringent reflectarray metasurface for beam engineering in infrared. Opt. Lett. 38, 462-464 (2013).

27. Nielsen, M. G., Pors, A., Albrektsen, O. \& Bozhevolnyi, S. I. Efficient absorption of visible radiation by gap plasmon resonators. Opt. Express 20, 13311-13319 (2012).

28. Johnson, P. B. \& Christy, R. W. Optical constants of the noble metals. Phys. Rev. B 6, 4370-4379 (1972).

\section{Acknowledgments}

We acknowledge financial support for this work from the Danish Council for Independent Research (the FTP project ANAP, contract no. 09-072949, and the FNU project, contract no. 12-124690).

\section{Author contributions}

S.I.B. conceived the idea, A.P. did the theoretical calculations and designed the samples. O.A. fabricated the structures, and I.P.R. performed the measurements. A.P. and S.I.B. wrote the manuscript based on input from all authors.

\section{Additional information}

Supplementary information accompanies this paper at http://www.nature.com/ scientificreports

Competing financial interests: The authors declare no competing financial interests.

How to cite this article: Pors, A., Albrektsen, O., Radko, I.P. \& Bozhevolnyi, S.I. Gap plasmon-based metasurfaces for total control of reflected light. Sci. Rep. 3, 2155; DOI:10.1038/srep02155 (2013)

This work is licensed under a Creative Commons AttributionNonCommercial-ShareAlike 3.0 Unported license. To view a copy of this license, visit http://creativecommons.org/licenses/by-nc-sa/3.0 